\title{
Anti-inflammatory effects of artesunate on atherosclerosis via miR-16-5p and TXNIP regulation of the NLRP3 inflammasome
}

\author{
Bo $\mathrm{Li}^{1,2}$, Zheqi Zhang ${ }^{2}$, Yili Fu ${ }^{1,3}$ \\ ${ }^{1}$ School of Life Science and Technology, Harbin Institute of Technology, Harbin, China; ${ }^{2}$ Department of Endocrinology, the Fourth Affiliated \\ Hospital of Harbin Medical University, Harbin, China; ${ }^{3}$ State Key Laboratory of Robotics and Systems, Harbin Institute of Technology, Harbin, \\ China \\ Contributions: (I) Conception and design: Y Fu, B Li; (II) Administrative support: Y Fu; (III) Provision of study materials or patients: B Li, Z Zhang; (IV) \\ Collection and assembly of data: B Li, Z Zhang; (V) Data analysis and interpretation: Y Fu, B Li; (VI) Manuscript writing: All authors; (VII) Final \\ approval of manuscript: All authors. \\ Correspondence to: Yili Fu. School of Life Science and Technology; State Key Laboratory of Robotics and Systems, Harbin Institute of Technology, 92 \\ West Dazhi Street, Nangang District, Harbin 150000, China. Email: meylfu@hit.edu.cn.
}

Background: Atherosclerosis (AS) is chronic inflammatory arterial disorder. Artesunate could exhibit antiinflammatory activity in AS, but its role in AS is still in its incipient stage. In this study, we explored the antiinflammatory effect of artesunate in AS and its underlying mechanism.

Methods: We isolated CD14 $4^{+}$monocytes from peripheral blood (PB) of 115 coronary heart disease (CHD) patients and 33 non-CHD patients confirmed by coronary angiography. Phorbol myristate acetate (PMA) was used to induce the differentiation of THP-1 monocytes to macrophages. Cells were treated with artesunate at a final concentration of $2.5,5$ or $10 \mu \mathrm{mol} / \mathrm{L}$. The activation of NLRP3 inflammasome was assessed by immunoblotting of apoptosis-associated speck-like protein containing caspase recruitment domain (ASC). The expression of pro-caspase-1/pro-interleukin (IL)-1ß/pro-IL-18 and their mature forms was measured using immunoblotting. A rat model of AS was induced by vitamin D3 (VD3) and a 21-day high-fat diet.

Results: Downregulated miR-16-5p and upregulated thioredoxin-interacting protein (TXNIP) was determined in $\mathrm{CD}_{14} 4^{+}$monocytes from CHD patients and associated with disease severity. Artesunate abrogated the activation of NLRP3 inflammasome in the presence of inflammasome activators in cultured macrophages. Artesunate reduced TXNIP expression and impaired the interaction between TXNIP and NLRP3, thereby inhibiting release of inflammatory cytokines and ASC production in cultured macrophages. In addition, miR-16-5p negatively regulated the messenger RNA (mRNA) of TXNIP. Artesunate increased the expression of miR-16-5p in a dose-dependent manner, and inhibition of miR-16-5p enhanced the secretion of inflammatory cytokines. Our in vivo experiments also demonstrated that artesunate reduced lipid accumulation, atherosclerotic plaque formation, and antagonized inflammation in a dose-dependent manner by upregulating miR-16-5p.

Conclusions: In summary, the present study unveiled a mechanism underlying the anti-inflammatory role of artesunate in AS.

Keywords: Artesunate; atherosclerosis (AS); microRNA-16-5p; LR family pyrin domain containing 3 (NLRP3); inflammasome

Submitted Aug 22, 2021. Accepted for publication Oct 16, 2021.

doi: 10.21037/atm-21-4939

View this article at: https://dx.doi.org/10.21037/atm-21-4939 


\section{Introduction}

The pathogenesis of atherosclerosis (AS) is not only involved in lipid accumulation, but also in immune cells and inflammation combined with hyperlipidemia, accompanied by elevated low-density lipoprotein (LDL) levels (1). Inflammation contributes to atherosclerotic plaque formation and rupture (2). Anti-inflammatory drugs or therapies have revealed avenues for AS treatment (3). Artesunate has been reported to exhibit an effect on inflammatory responses and immunosuppression as a water-soluble derivative from artemisinin, a traditional Chinese herb (4). Artesunate could attenuate atherosclerotic lesion formation alone or in combination with rosuvastatin via repression of pro-inflammatory cytokines [tumor necrosis factor- $\alpha$ (TNF- $\alpha$ ) and interleukin-6 (IL-6)] and pro-inflammatory chemokines (IL-8) (5). However, the mechanism of artesunate in AS has remained enigmatic.

The LR family pyrin domain containing 3 (NLRP3) inflammasome is composed of NLRP3 (cytoplasmic sensor molecule), apoptosis-associated speck-like protein containing caspase recruitment domain (ASC; adaptor protein), and pro-caspase-1 (effector protein) (6). The NLRP3 inflammasome pathway was proposed to be suppressed by artesunate thereby alleviating glomerular mesangial cell injury (7). The inhibitory effect of artesunate on AS by upregulation of LPL expression via KLF2/ NRF2/TCF7L2 axis (8). NLRP3 inflammasome activation promoted the development of AS (9). In the present study, we sought to determine the alleviating effect of artesunate on AS in regulation of NLRP3 inflammasome and its possible downstream mechanism. TXNIP repression was reported to inactivate NLRP3 inflammasome, and attenuate inflammation. As an $\alpha$-arrestin protein, TXNIP has been implicated in multiple pleiotropic biological responses (10). For example, TXNIP has been shown to mediate inflammatory responses by blocking the activity of thioredoxin (11). Elevated expression of TXNIP was observed in AS and its inhibition promoted proliferation of endothelial cells XNIP was predicted to be a target gene of microRNA (miR)-16-5p in the TargetScan database (http://www.targetscan.org/). miRNAs have been proposed to regulate multiple developmental and cellular procedures with the length of approximately 21 nucleotides (12). Multiple miRNAs have been implicated in AS, including miR-122 (13) and miR-24 (14); miR-16-5p was downregulated in diabetic kidney disease compared to type 1 diabetes mellitus verified by bioinformatic analysis, and miR-16-5p could also modulate genes from AS (15).
The above-mentioned findings unveil a possible mechanism underlying the anti-inflammatory effect of artesunate by blocking TXNIP-dependent NLRP3 inflammasome activation by regulating miR-16-5p in AS. Thus, in this study, we extracted $\mathrm{CD} 14^{+}$monocytes from peripheral blood (PB) of coronary heart disease (CHD) patients and 33 non-CHD patients, used phorbol myristate acetate (PMA)induced human monocyte cells, and finally established a rat model to elucidate the anti-inflammatory role of artesunate in AS. We present the following article in accordance with the ARRIVE reporting checklist (available at https://dx.doi. org/10.21037/atm-21-4939).

\section{Methods}

\section{Ethical approval}

The study was approved by the Ethics Committee in the Fourth Affiliated Hospital of Harbin Medical University and conformed to the tenets of the Declaration of Helsinki (as revised in 2013). Each individual provided written informed consent prior to their participation. Animal experiments were approved by Animal ethics committee of the Fourth Affiliated Hospital of Harbin Medical University for the care and use of animals. The study was also conducted in strict accordance with the standard of the Guide for the Care and Use of Laboratory Animals issued by the National Institutes of Health.

\section{Participants}

Patients who underwent coronary angiography (CAG) from June 2006 to December 2018 in the Fourth Affiliated Hospital of Harbin Medical University were recruited to the study, including a total of $115 \mathrm{CHD}$ patients with at least one coronary artery stenosis $>50 \%$, and 33 nonCHD patients (control). All participants received no prior treatment of statins and other lipid lowering drugs, and had no history of valvular heart disease, malignant arrhythmia, acute infection, and severe liver or renal dysfunction before they were enrolled. The severity of CHD was assessed by Gensini score (16).

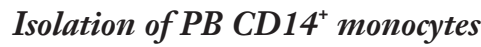

The PB of CHD participants (about $20 \mathrm{~mL}$ ) was extracted, mixed with $2 \mathrm{~mL}$ heparin for anticoagulation, and human lymphocyte separation solution was used to obtain monocytes using density gradient centrifugation; the 
human $\mathrm{CD}_{14}{ }^{+}$cell isolation kit (Miltenyi Biotec, Bergisch Gladbach, Germany) was adopted to isolate $\mathrm{CD} 14^{+}$cells adhering to the manufacturer's instructions. A heart puncture was performed on the rat hearts using heparin syringe and then blood samples $(5 \mathrm{~mL})$ were taken. The monocytes were obtained using rat lymphocyte separation solution by density gradient centrifugation; the rat CD14 ${ }^{+}$ cell isolation kit (Miltenyi Biotec) was used to sort rat CD $14^{+}$cells strictly according to the manufacturer's instructions. Flow cytometry staining was performed using CD14-fluorescein isothiocyanate (FITC) (eBioscience, San Diego, CA, USA). Flow cytometry (Becton, Dickinson, and Co., Franklin Lakes, NJ, USA) was finally adopted to assess the purity of the sorted $\mathrm{CD} 14^{+}$cells and purity of $>95 \%$ was regarded as $\mathrm{CD} 14^{+}$cells.

\section{Enzyme-linked immunosorbent assay}

The plasma of participants and rats or THP1 cells were adopted to culture supernatants, which were then subjected to an enzyme-linked immunosorbent assay (ELISA) kit of interleukin (IL)-1 $\beta$, IL-18, total cholesterol (TC), triglyceride (TG), high-density lipoprotein (HDL), and LDL separately. The kits were obtained from Wuhan Beinglay Biotechnology Co., Ltd. (Wuhan, Hubei, China) and Shanghai Yuanye Biotechnology Co., Ltd. (Shanghai, China), and performed according to the instructions when the concentration of substance was positively correlated with the color of the final product. The microplate reader (Biotek, Winooski, VT, USA) was set to measure the optical density $(\mathrm{OD})$ at the corresponding wavelength and calculate the concentration of the substance.

\section{Reverse transcription quantitative polymerase chain reaction}

Total RNA of CD14 cell monocytes of people or rat was extracted using TRIzol (Invitrogen, Carlsbad, CA, USA) and RNA concentration was assessed using Nanodrop 2000 (Thermo Fisher Scientific, Waltham, MA, USA). Then, complementary DNA (cDNA) was synthesized, and antisense miRNA was used in a PCR kit (Thermo Fisher Scientific). Reverse transcription quantitative polymerase chain reaction (RT-qPCR) was then performed with ABI7500 qPCR kit (Thermo Fisher Scientific) with beta $(\beta)$-actin or U6 as internal references. The ratio of the gene expression of observation to control was calculated by means of relative quantification $\left(2^{-\Delta \Delta \mathrm{Ct}}\right.$ method). Primer sequences are shown in Table S1. Primers were provided by Genepharma (Shanghai, China).

\section{Dual luciferase reporter gene assay}

The bioinformatic prediction site predicted the possible target gene of miR-16-5p, which was validated by dual luciferase reporter gene assay. The synthetic TXNIP 3'-untranslated region (3'UTR) gene fragment was introduced into pMIR-reporter (Promega, Madison, WI, USA) using the endonuclease sites SpeI and Hind III, and the complementary sequence mutated (MUT) sites of the seed sequence were designed on the Cxcl12 wild type (WT). Following endonuclease digestion, the WT and MUT target fragments were separately inserted into the pMIR-reporter reporter plasmid using T4 DNA ligase. The correctly sequenced WT and MUT luciferase reporter plasmids were co-transfected with miR-16-5p mimic and mimic-NC to the cell HEK-293T (Shanghai Beinuo Biology, Shanghai, China). Following 48 h-transfection, the cells were collected and lysed, centrifuged for 3-5 min, and the supernatant was taken. The luciferase activity in the cell extract was assessed using a luciferase assay kit (DualLuciferase Reporter Assay System, Promega, USA): $100 \mu \mathrm{L}$ LAR II and $20 \mu \mathrm{L}$ cell lysate were added to the measuring tube to measure the target fluorescence, and $100 \mu \mathrm{L}$ Stop \& Glo Reagent (Promega) was added to measure the fluorescence of internal reference. The relative luciferase activity was assessed by the value of target/internal fluorescence with a fluorescence detector (Promega).

\section{Cell culture and treatment}

Human monocyte cell line THP1 was obtained from Shanghai Institute for Biological Sciences (Shanghai, China). The THP1 cells were developed in a $37^{\circ} \mathrm{C}, 5 \%$ $\mathrm{CO}_{2}$ cell culture incubator with Roswell Park Memorial Institute (RPMI) 1640 Petri (Gibco, Carlsbad, CA, USA) containing $10 \% 65^{\circ} \mathrm{C}$ heated-inactivated fetal bovine serum (FBS; Gibco). At a final concentration of $5 \mathrm{ng} / \mathrm{mL}$, PMA (Sigma Aldrich, St. Louis, MO, USA) was adopted to induce differentiation of monocyte (THP1 cell) to macrophage.

Plasmids containing miR-16-5 p mimic, mimic-NC, inhibitor-NC, and miR-16-5p inhibitors (Shanghai Rainbow Co., Ltd., Shanghai, China) were transfected into THP1-induced macrophages using Lipofectamine 2000 (Invitrogen) according to the manufacturer's instructions. Cells were harvested $48 \mathrm{~h}$ after transfection for subsequent 
experiments.

Cells were treated with artesunate at a final concentration of $2.5,5$, or $10 \mu \mathrm{mol} / \mathrm{L}$, Artesunate at a concentration of $10 \mu \mathrm{mol} / \mathrm{L}$, artesunate was used to treat cells in the presence of activators of NLRP3 inflammasome adenosine triphosphate (ATP; Sigma Aldrich; $5 \mathrm{mM}, 2 \mathrm{~h}$ ), Nigericin ( $\mu \mathrm{M}, 2 \mathrm{~h})$, Alum $(50 \mu \mathrm{g} / \mathrm{mL} ; 4 \mathrm{~h})$, and monosodium urate (MSU; InvivoGen, San Diego, CA, USA; $100 \mu \mathrm{mol} / \mathrm{mL} ; 4 \mathrm{~h}$ ).

\section{Immunoblotting}

Total protein of THP1-induced differentiation of macrophages was extracted using protein strong lysate (Beyotime Biotechnology, Shanghai, China). Protein samples were separated by $10 \%$ polyacrylamide gel electrophoresis (PAGE) and then transferred onto polyvinylidene fluoride (PVDF) membranes (Millipore, Billerica, MA, USA). The membrane was probed with primary antibodies overnight at $4{ }^{\circ} \mathrm{C}$, then reprobed with secondary antibody horseradish peroxidase-labeled goat anti-mouse (1:10,000, ab6789, Abcam) or goat anti-rabbit (1:10,000, ab6721, Abcam) for incubation for $1 \mathrm{~h}$ at room temperature using enhanced chemiluminescence (ECL) detection reagents. The grey value of bands was analyzed using ImageJ Plus 6.0 software (Media Cybernetics, Silver Spring, MD, USA). Relative protein expression was analyzed by the ratio of gray value of target band to that of internal reference. Primary antibodies were as follows, antirabbit IL-1 $\beta$ polyclonal antibody $(1: 1,000$, ab2105, Abcam, Cambridge, UK), anti-mouse mature IL- $1 \beta$ monoclonal antibody (1:500, Asp116, Cell Signaling Technology, Beverly, MA, USA), anti- rabbit-TXNIP monoclonal antibody (1:1,000, ab188865, Abcam), anti-rabbit NLRP3 polyclonal antibody (1:500, ab2 14185, Abcam), antirabbit recombinant caspase- 1 monoclonal antibody (1:1,000, ab207802, Abcam), anti-rabbit IL-18 polyclonal antibody (1:1,000, ab191152, Abcam), anti-mouse mature IL-18 monoclonal antibody (1:1,000, D044-3M2, MBL international corporation, Woburn, UK), and anti-mouse $\beta$-actin monoclonal antibody (1:5,000, ab8225, Abcam).

\section{ASC}

Macrophages treated with inflammasome activators, artesunate, miR-16-5p-mimic, or miR-16-5p-inhibitor were collected. Freshly prepared sodium dodecyl-sulphate (SDS) lysis buffer was lysed on ice for $10 \mathrm{~min}$, and $4 \mathrm{mM}$ dextran sulfate sodium (Sigma-Aldrich Chemical Company,
St. Louis MO, USA) was added to decrosslink at room temperature for $40 \mathrm{~min}$. The supernatant was discarded following centrifugation, and then SDS buffer was added. The anti-mouse ASC monoclonal antibody (1:500, sc271054, Santa Cruz Bio., Santa Cruz, CA, USA) was used for ASC production using immunoblotting.

\section{Coimmunoprecipitation}

The coimmunoprecipitation (CO-IP) assay was performed using a Thermo IP kit strictly according to the manufacturer's instructions: IP lysis wash buffer supplemented with protease inhibitor was used to lyse macrophages treated with ATP and/or $10 \mu \mathrm{mol} / \mathrm{L}$ artesunate, and then macrophages were incubated with the cells $(5 \mathrm{~min})$ on ice and mixed. Following centrifugation (13,000 g; $10 \mathrm{~min})$, supernatants were collected and a part of it was used for input. Following pretreatment of the cell lysis, it was divided into 3 parts and then added to the spin columns. The cell lysis was added with TXNIP and anti-immunoglobulin $\mathrm{G}$ ( $\mathrm{IgG}$ ) antibody, followed by incubation overnight at $4{ }^{\circ} \mathrm{C}$, and then combined with Pierce protein $\mathrm{A} / \mathrm{G}$ agarose for $2 \mathrm{~h}$. Then, the mixture was centrifuged (1,000 g; $1 \mathrm{~min}$ ), waste solution was discarded, and the resin was washed with IP lysis wash buffer (thrice), using conditional buffer washing (twice) for antigen elution, which was then subjected to immunoblotting to detect the interaction between TXNIP and NLRP3.

\section{Establishment of a rat model of coronary $A S$}

Male Sprague Dawley rats (7-8 weeks old, body weight 180-240 g) were purchased from Shanghai Research Institute of Pharmaceutical Industry (Shanghai, China). Briefly, 60 rats were intraperitoneally injected with vitamin D3 (VD3) once in a day for $3 \mathrm{~d}$ at a total dose of $7 \mathrm{WU} / \mathrm{Kg}$ and fed a high-fat diet (20 g) for $21 \mathrm{~d}(20 \mathrm{~g}$; basal feed, $3.5 \%$ cholesterol, $10 \%$ lard, $0.2 \%$ propyl thiouracil, $0.5 \%$ sodium cholate, and $5 \%$ white sugar). A rat model of AS was then established as previously reported (17). The AS rats were intragastrically (i.g.) administered at the dosage of $10 \mathrm{mg} / \mathrm{kg} / \mathrm{d}$ (10 rats), $30 \mathrm{mg} / \mathrm{kg} / \mathrm{d}$ (10 rats), and $50 \mathrm{mg} / \mathrm{kg} / \mathrm{d}$ (30 rats), respectively. Artesunate was supplemented with $0.3 \% \mathrm{NaHCO}_{3}$ (Guilin Pharmaceutical Factory, Guangxi, China). The remaining 10 AS rats were administered (i.g.) at $0.3 \% \mathrm{NaHCO}_{3}$ for $7 \mathrm{~d}$ in the absence of artesunate. The remaining rats were intraperitoneally injected with an equal volume of saline $(20 \mathrm{~g} ; 7 \mathrm{WU} / \mathrm{Kg}$ ) for $21 \mathrm{~d}$. A total of 20 
rats treated with $50 \mathrm{mg} / \mathrm{kg} / \mathrm{d}$ artesunate were injected with antagomir-16-5p-NC and antagomir-16-5p (Shanghai Genepharm Co., Ltd., Shanghai, China) via the tail vein $(80 \mathrm{mg} / \mathrm{kg} / \mathrm{d})$ for $3 \mathrm{~d}$. These rats were euthanized by cervical dislocation.

\section{Hematoxylin-eosin staining}

The aorta tissues of rats were subjected to hematoxylineosin (HE) staining. Briefly, tissues were dehydrated and dewaxed. The sections were treated with eosin (Beyotime, Shanghai, China) and then mounted using neutral gum or Canadian gum. A light microscope (Leica, DMI3000, Wetzlar, Germany) was adopted to observe the inflammation of the brain, spleen, and liver tissue and the area of aortic AS plaque was calculated.

\section{Statistical analysis}

All data, representative of 3 independent experiments in triplicate, are shown as mean \pm standard deviation and analyzed by SPSS 21.0 software (IBM Corp., Armonk, NY, USA), with $\mathrm{P}<0.05$ as a level of statistical significance. If the data conformed to normal distribution and homogeneity of variance, unpaired $t$-test was adopted to analyze the differences between 2 groups and one-way analysis of variance (ANOVA) with Tukey's test was utilized to compare data among multiple groups. Pearson's correlation analysis was used to analyze correlations between indicators. A $\mathrm{P}$ value $<0.05$ was considered statistically significant.

\section{Results}

\section{Downregulated miR-16-5p and upregulated TXNIP was associated with disease severity of $A S$}

Statistical analysis was performed on the general data of the CHD and the non-CHD patients, and the results revealed that there was no obvious difference in gender, systolic blood pressure, diastolic blood pressure, age, creatinine, heart rate, and alanine aminotransferase (ALT) in the CHD patients and non-CHD patients $(\mathrm{P}>0.05)$. As shown in Figure 1A, ELISA results identified a remarkable rise in the protein level of IL- $1 \beta$ and IL-18 in PB of CHD patients compared to that of non-CHD patients $(\mathrm{P}<0.05)$. Pearson's correlation analysis (Figure $1 B$ ) was conducted between Gensini score and protein level of IL-1 $\beta$ and IL18 in $\mathrm{PB}$, which revealed that Gensini score was positively correlated with protein level of IL-1 $\beta(\mathrm{r}=0.779, \mathrm{P}<0.05)$ and IL-18 $(r=0.534, \mathrm{P}<0.05)$ in PB. The results of RTqPCR (Figure 1C) displayed that compared to non-CHD patients, the expression of miR-16-5p in $\mathrm{PB}$ monocytes strikingly dropped, while the mRNA expression of TXNIP potently rose $(\mathrm{P}<0.05)$. Pearson's correlation analysis (Figure 1D) was performed between the Gensini score in CHD patients and the expression of miR-16-5p and TXNIP in PB monocytes. Results revealed that Gensini score and the expression of miR-16-5p $(\mathrm{r}=-0.557, \mathrm{P}<0.05)$ or TXNIP mRNA expression $(r=0.727, \mathrm{P}<0.05)$ in $\mathrm{PB}$ monocytes were correlated, and the expression of miR-16-5p was negatively correlated with TXNIP mRNA expression $(r=-0.438$, $\mathrm{P}<0.05)$. Taken together, the rising protein levels of IL- $1 \beta$ and IL-18 in CD14 $4^{+}$monocytes were indicative of severity of coronary AS. Also, the dropped expression of miR-16$5 \mathrm{p}$ and elevated TXNIP mRNA expression in PB CD14 monocytes are indicators of severity of coronary AS.

\section{TXNIP was the target of miR-16-5p}

The TargetScan database (Figure $2 A$ ) predicted a binding site between miR-16-5p and the 3'UTR of TXNIP, suggesting that TXNIP may be negatively regulated by miR-16-5p. The results of the dual luciferase reporter gene assay (Figure $2 B$ ) validated that the luciferase activity of the TXNIP WT 3'UTR strikingly reduced in the presence of miR-16-5p-mimic $(\mathrm{P}<0.05)$ relative to the TXNIP MUT 3'UTR (P>0.05). Next, we conducted gainor loss- function assay of miR-16-5p in THP1 cells using miR-16-5p mimic and inhibitor and adopted RT-qPCR (Figure 2C) to assess the expression of $\mathrm{miR}-16-5 \mathrm{p}$ and TXNIP. The results revealed increased miR-16-5p expression and reduced TXNIP mRNA expression in THP1 cells in response to miR-16-5p mimic compared to inhibitor-NC, while an opposite trend was seen in response to miR-16-5p inhibitor compared to inhibitor- $\mathrm{NC}(\mathrm{P}<0.05)$.

\section{Artesunate inbibited release of pro-inflammatory factors activated by NLRP3 inflammasome in macrophages}

To detect the anti-inflammatory effect of artesunate, we employed ATP to activate NLRP3 inflammasome and treated cells with artesunate at different concentrations in PMA-induced macrophages. The results of ELISA (Figure $3 A$ ) and immunoblotting (Figure $3 B$ ) identified elevated levels of IL-1 $\beta$ and IL-18, elevated expression levels of pro-caspase-1, -IL-1 $\beta$, and -IL-18 and their 

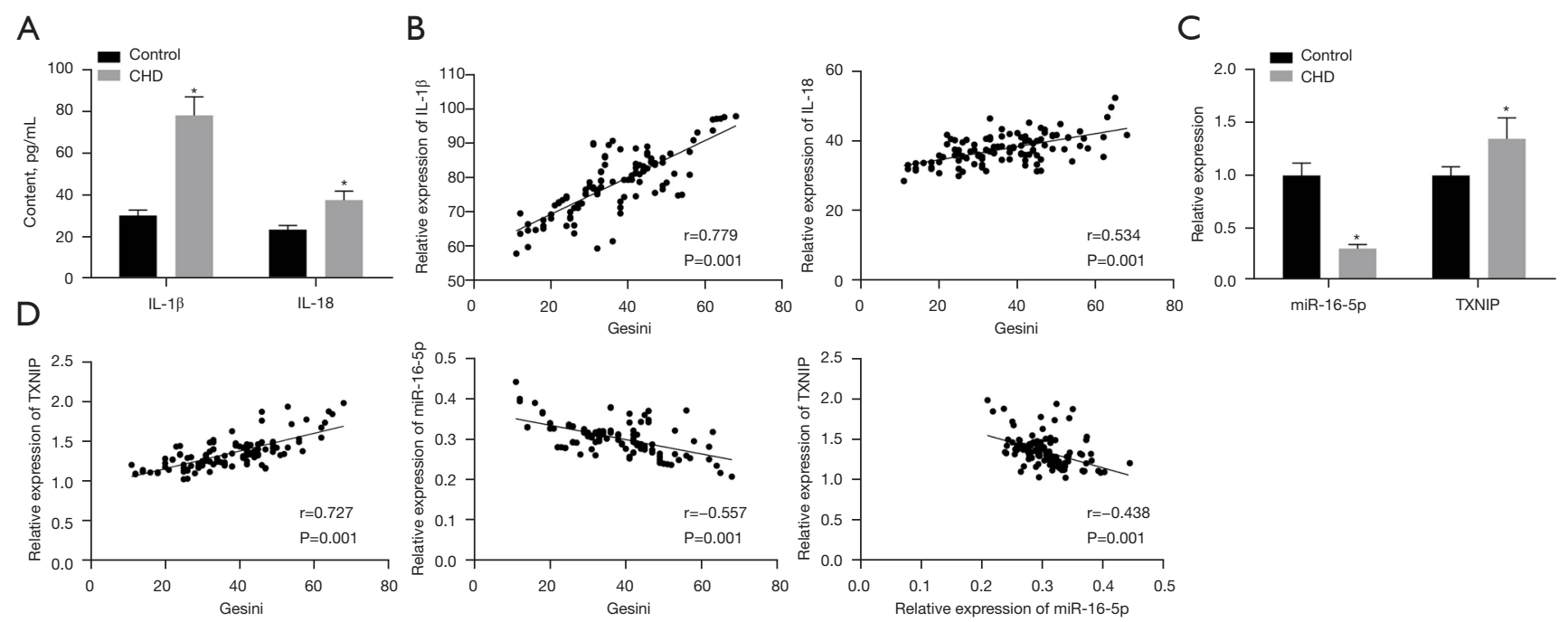

Figure 1 Reduced expression of miR-16-5p and elevated expression of TXNIP in PB are indicative of severity of coronary AS. CD14 monocytes were isolated from $\mathrm{PB}$ of $115 \mathrm{CHD}$ patients and 33 non-CHD patients confirmed by coronary angiography. (A) The protein level of IL-1 $\beta$ and IL-18 in CD14 ${ }^{+}$monocytes assessed by ELISA. (B) Pearson's correlation analysis between Gensini scores and protein level of IL-1 $\beta$ and IL-18 in CD14 ${ }^{+}$monocytes. (C) The expression of miR-16-5p and TXNIP in CD14 ${ }^{+}$monocytes determined by RT-qPCR. (D) Pearson's correlation analysis between the Gensini scores and the expression of miR-16-5p and TXNIP in CD14 ${ }^{+}$monocytes. * (relative to non-CHD patients), indicates $\mathrm{P}<0.05$ by unpaired $t$-test. $\mathrm{PB}$, peripheral blood; CHD, coronary heart disease; IL-1 $\beta$, interleukin-1 $\beta$; ELISA, enzyme-linked immunosorbent assay; RT-qPCR, reverse transcription quantitative polymerase chain reaction.
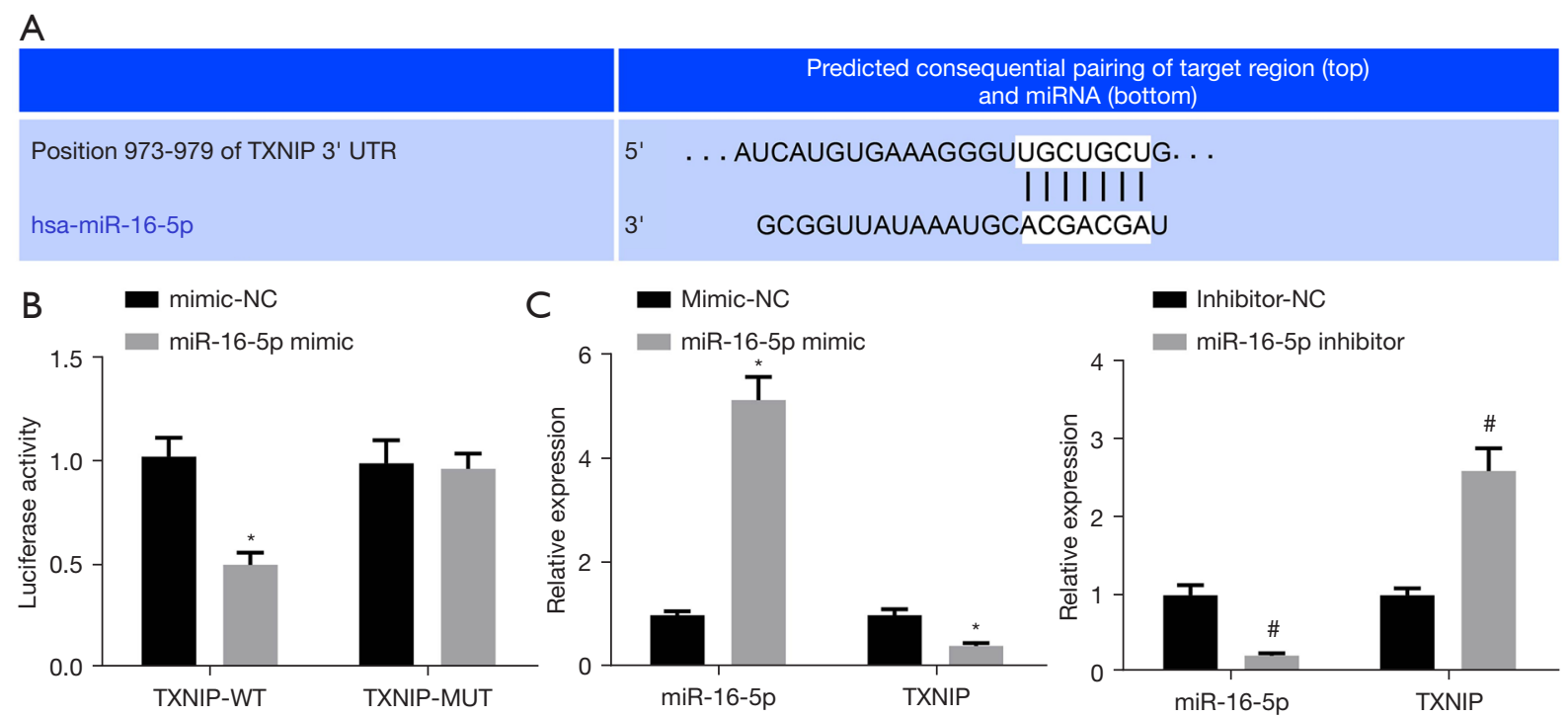

Figure 2 TXNIP is the target gene of miR-16-5p. Cells were transfected with miR-16-5p-mimic or mimic-NC. (A) Possible binding site between miR-16-5p and TXNIP in the TargetScan database. (B) Interaction between miR-16-5p and TXNIP validated by dual luciferase reporter gene assay; * (relative to mimic NC + TXNIP-3'UTR WT), indicates $\mathrm{P}<0.05$ by unpaired $t$-test. (C) The mRNA expression of TXNIP in THP1 cells assessed by RT-qPCR; * (relative to mimic-NC), and \# (relative to inhibitor-NC), indicate $\mathrm{P}<0.05$ by unpaired $t$-test. mRNA, messenger RNA. 

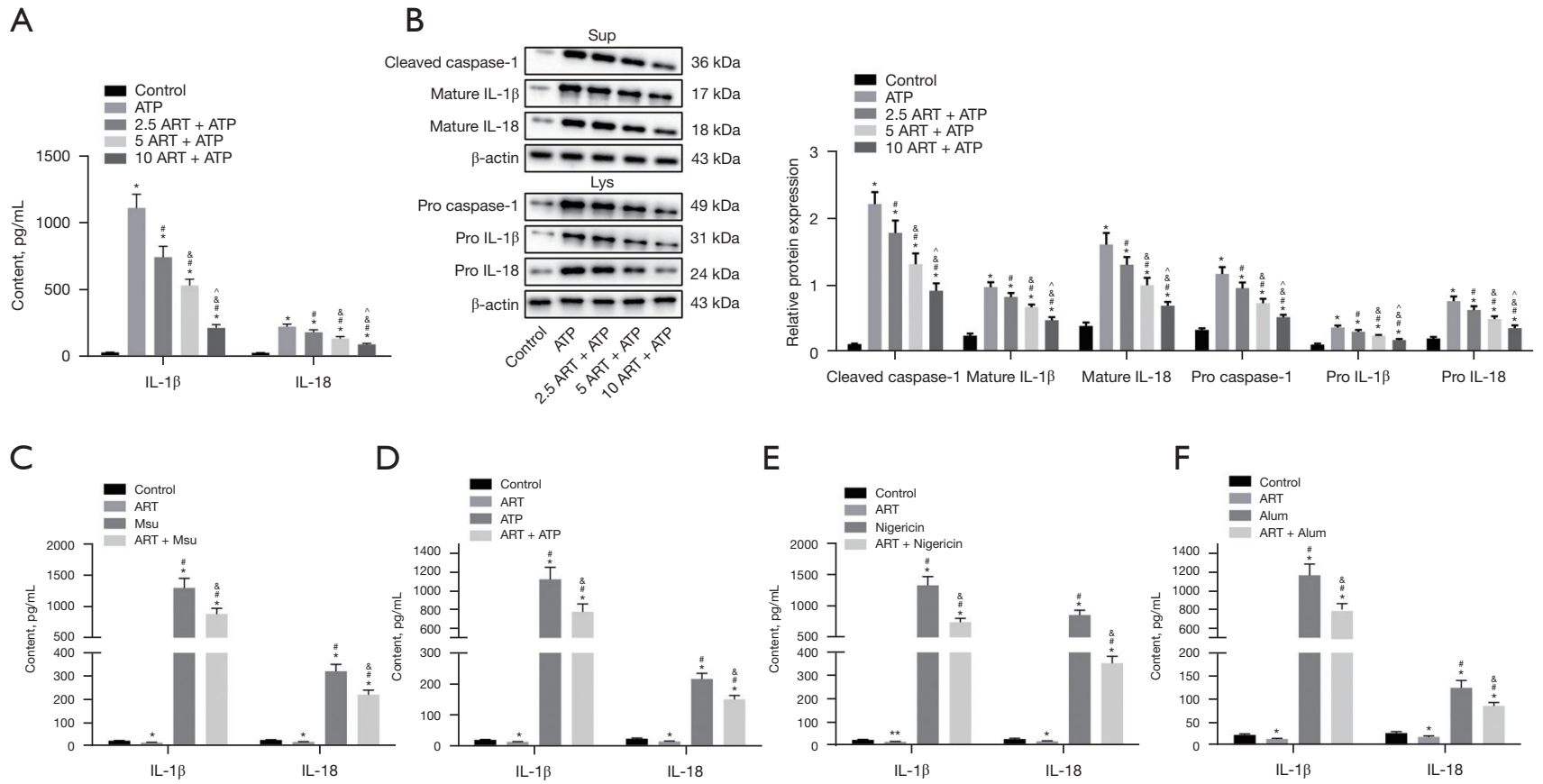

G

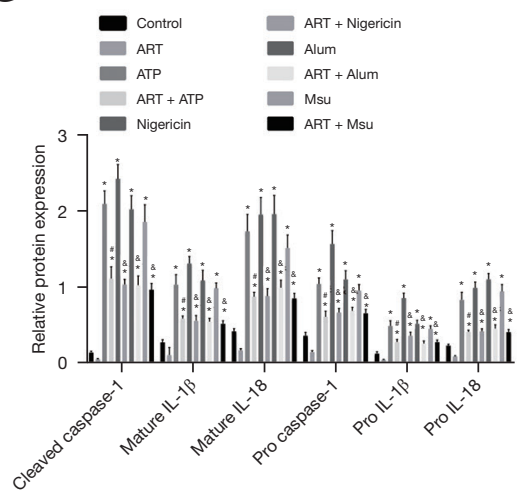

$\mathrm{H}$

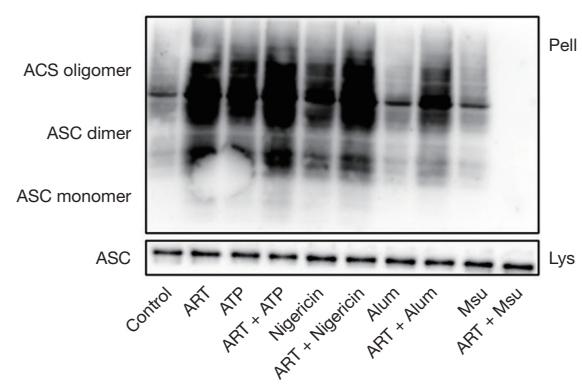

Figure 3 Artesunate inhibits secretion of pro-inflammatory factors activated by NLRP3 inflammasome in macrophages. (A,C-F) The expression of IL-1 $\beta$ and IL-18 in macrophage culture supernatant assessed by ELISA. (B,G) The expression of pro-caspase-1, -IL-1 $\beta$ and -IL-18 and their mature forms released in macrophages assessed by immunoblotting. $(\mathrm{H})$ ASC production in macrophages assessed by ASC. * (relative to control), ${ }^{*}$ (relative to ATP), ${ }^{\&}$ (relative to AS + low-dose artesunate) and ${ }^{\wedge}$ (relative to AS + medium-dose artesunate), indicates $\mathrm{P}<0.05$ in panel (A); * (relative to control), ${ }^{*}$ (relative to artesunate) and ${ }^{*}$ (relative to ATP, Nigericin, Alum, or Msu), indicates $\mathrm{P}<0.05$ in panel (C-F). Statistical significance was confirmed by one-way ANOVA/Tukey's test. IL-1 $\beta$, interleukin-1 $\beta$; ELISA, enzyme-linked immunosorbent assay; ASC, apoptosis-associated speck-like protein containing caspase recruitment domain; ATP, adenosine triphosphate; Msu, monosodium urate.

mature forms (caspase-1, mature IL-1 $\beta$, and mature IL-18) released in macrophages in response to ATP treatment. As expected, we found artesunate treatment downregulated levels of IL-1 $\beta$ and IL-18, reduced the expression levels pro-caspase-1, -IL-1 $\beta$, and -IL-18 and their mature forms secreted from macrophages at a dose-dependent manner.
In the subsequent experiments, we activated NLRP3 inflammasome in the presence of ATP, Nigericin, Alum, and $\mathrm{Msu}$ with or without $10 \mu \mathrm{mol} / \mathrm{L}$ artesunate treatment. The ELISA (Figure $3 C-3 F$ ) and immunoblotting results (Figure $3 G, 3 H$ ) displayed that NLRP3 inflammasome could be activated in the presence of ATP, Nigericin, Alum, 
and Msu in PMA-induced THP1 cells, as evidenced by upregulated levels of IL-1 $\beta$ and IL-18, higher expression levels of pro-caspase-1, -IL-1 $\beta$, and -IL-18 and their mature forms released in macrophages as well as more ASC production in cultured macrophages $(\mathrm{P}<0.05)$. In addition, repressed levels of IL-1 $\beta$ and IL-18, pro-caspase-1, -IL-1 $\beta$, and -IL-18 and their mature forms released in macrophages as well as less ASC generation in macrophages were observed in cells treated with $10 \mu \mathrm{mol} / \mathrm{L}$ artesunate in the presence of ATP, Nigericin, Alum, or Msu. Taken together, ATP, Nigericin, Alum, and Msu contributed to elevated expression of caspase-1, IL-1 $\beta$, and IL-18 and more ASC production, while artesunate treatment could reverse the trend.

\section{Artesunate regulates TXNIP-mediated activation of NLRP3 inflammasome}

As confirmed above, we found that artesunate inhibited NLRP3 inflammasome. To better explore the underlying mechanism of artesunate on NLRP3 inflammasome, we adopted immunoblotting and CO-IP assay. As shown in Figure $4 A, 4 B$, results revealed that the protein expression of TXNIP exhibited no apparent change in response to ATP compared to control $(\mathrm{P}>0.05)$, while the interaction between TXNIP and NLRP3 was enhanced $(\mathrm{P}<0.05)$. Results also displayed that artesunate treatment also reduced the protein expression of TXNIP and the interaction between TXNIP and NLRP3 $(\mathrm{P}<0.05)$. The results of ELISA (Figure $4 C)$ demonstrated that artesunate treatment repressed levels of IL- $1 \beta$ and IL-18 in macrophage supernatant. Taken together, artesunate reduced the protein expression of TXNIP and the interaction between TXNIP and NLRP3, thereby inhibiting secretion of IL-1 $\beta$ and IL-18 in macrophages.

\section{Artesunate deactivated the NLRP3 inflammasome by targeted inbibition of TXNIP by miR-16-5p deactivating}

To better elucidate the mechanism underlying the influence of artesunate on TXNIP and NLRP3 inflammasome, we elevated the expression of miR-16-5p in macrophages using miR-16-5p mimic followed by ATP treatment. Macrophages treated with miR-mimic NC followed by phosphatebuffered saline (PBS) treatment served as controls. The RTqPCR (Figure 5A), ELISA (Figure 5B), and immunoblotting (Figure 5C,5D) results demonstrated that the expression of miR-16-5p and TXNIP exhibited no marked difference
$(\mathrm{P}>0.05)$ in macrophages treated with ATP or PBS following miR-mimic; ATP treatment strikingly elevated the expression of miR-16-5p, but downregulated expression of TNXIP, IL-1 $\beta$, and IL-18, repressed pro-caspase-1, -IL-1 $\beta$, and -IL-18 and their mature forms released in macrophages and less ASC generation were witnessed following miR-16$5 \mathrm{p}$ overexpression $(\mathrm{P}<0.05)$. Next, we adopted RT-qPCR (Figure 5E) to assess the expression of miR-16-5p in THP1differentiated macrophages treated with artesunate at different concentrations. The results displayed that the artesunate treatment markedly elevated the expression of miR-16-5p in a dose-dependent manner $(\mathrm{P}<0.05)$. Subsequently, we repressed miR-16-5p in THP1-differentiated macrophages, followed by treatment with $10 \mu \mathrm{mol} / \mathrm{L}$ artesunate, and then adopted RT-qPCR (Figure 5F), ELISA (Figure $5 G$ ), and immunoblotting (Figure $5 \mathrm{H}, 5 \mathrm{I}$ ). No significant difference in the expression of miR-16-5p was observed between macrophages treated with PBS or ATP followed by inhibitor-NC $(\mathrm{P}>0.05)$, while the presence of artesunate upregulated the expression of miR-16-5p. Either ATP or artesunate treatment markedly upregulated the expression of IL-1 $\beta$ and IL-18, expression of pro-caspase-1, -IL-1 $\beta$, and $-\mathrm{IL}-18$ and their mature forms released in macrophages and produced more ASC, but downregulated the expression of TXNIP in macrophages treated inhibitor-NC relative to PBS $(\mathrm{P}<0.05)$. In addition, artesunate treatment strikingly elevated expression of miR-16-5p, but repressed expression of TXNIP, reduced expression of IL- $1 \beta$, expression of IL18, pro-caspase-1, -IL-1 $\beta$, and -IL-18 and their mature forms released in macrophages and produced less ASC production in macrophages treated with inhibitor-NC and ATP $(\mathrm{P}<0.05)$. The results were confirmed by the addition of miR-16-5p inhibitor instead of miR-16-5p mimic. Taken together, artesunate inhibited miR-16-5p-mediated TXNIP, thereby inactivating the NLRP3 inflammasome.

\section{Artesunate inhibits atherosclerotic plaque formation by its anti-inflammatory effects in vivo}

A rat model of coronary AS was established and then treated with artesunate at low dose $(10 \mathrm{mg} / \mathrm{kg} / \mathrm{d})$, medium dose $(30 \mathrm{mg} / \mathrm{kg} / \mathrm{d})$, or high dose $(50 \mathrm{mg} / \mathrm{kg} / \mathrm{d})$. Rats injected with saline $3 \mathrm{~d}+$ regular diet $21 \mathrm{~d}+0.3 \% \mathrm{NaHCO}_{3}$ served as control. Meanwhile, to better elucidate the mechanism of artesunate on miR-16-5p, we interfered miR-16$5 \mathrm{p}$ in AS rats treated with high-dose artesunate using antagomir-16-5p. We adopted HE staining (Figure 6A) to detect the plaque formation in aorta of rats and the results 
A

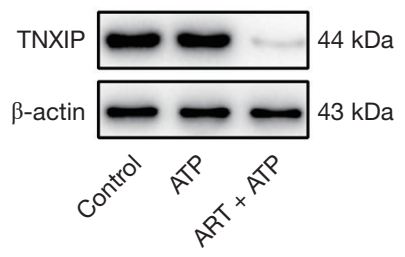

B

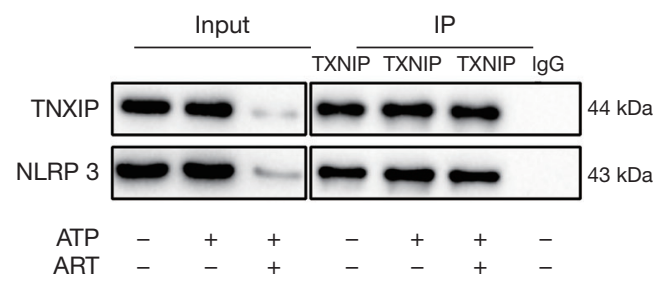

C

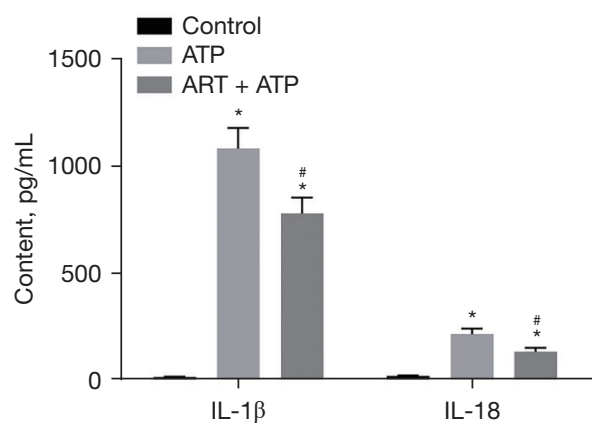

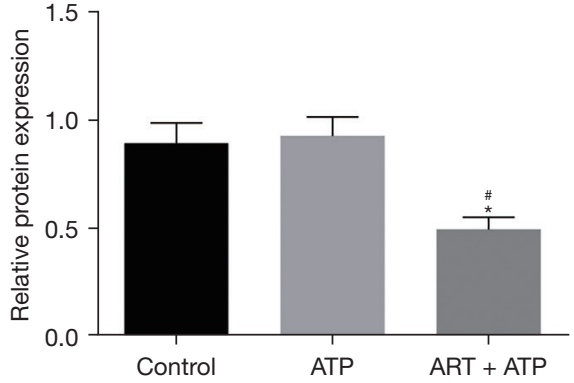

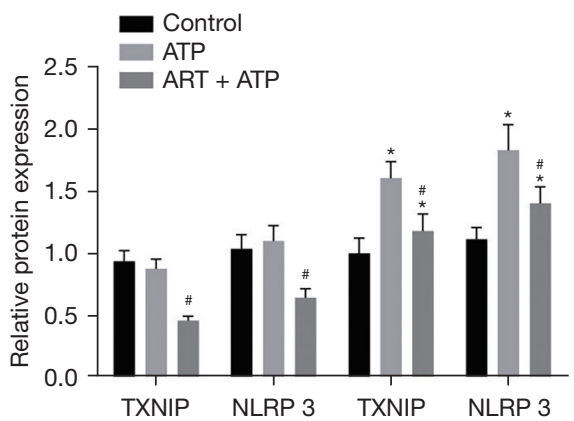



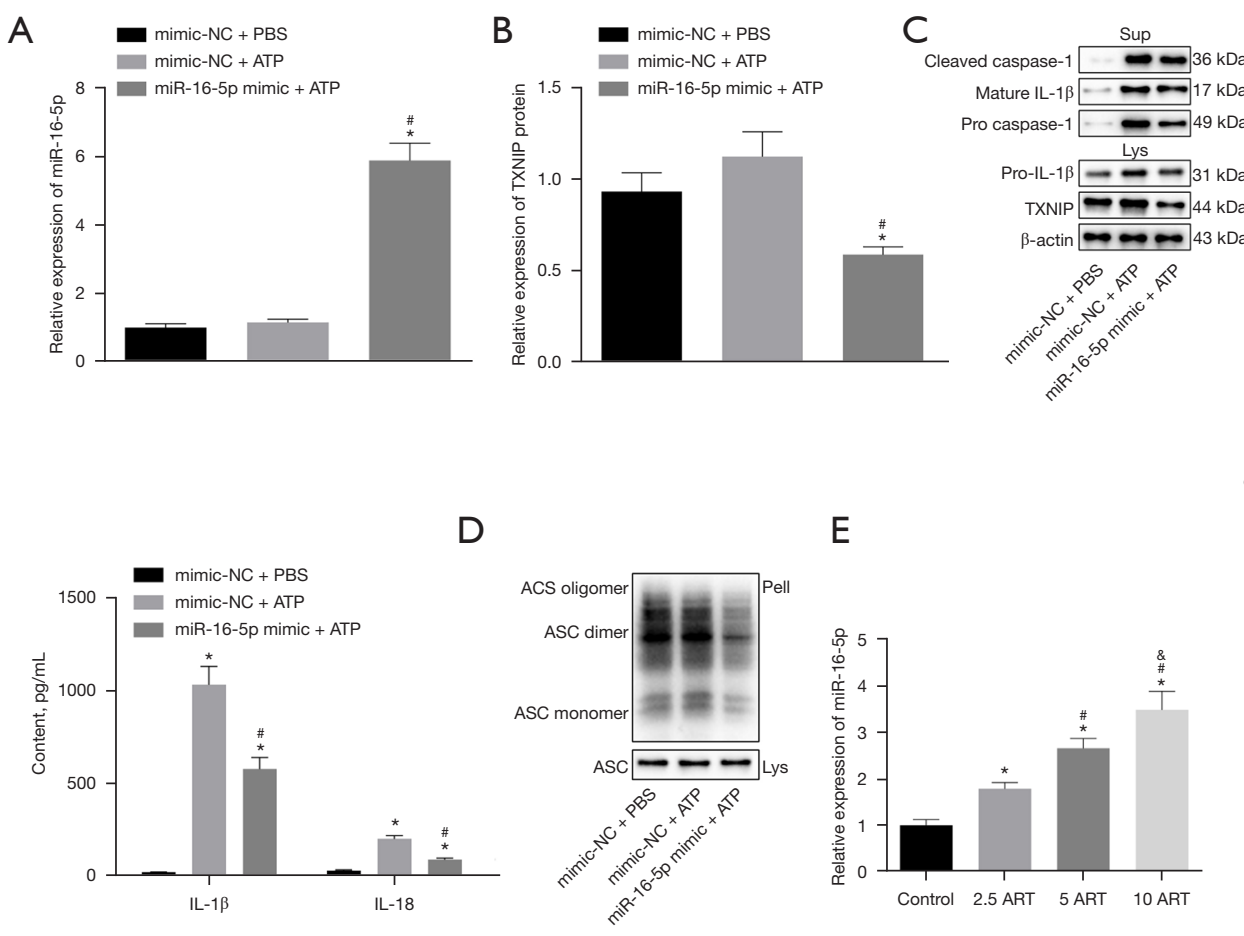

$E$

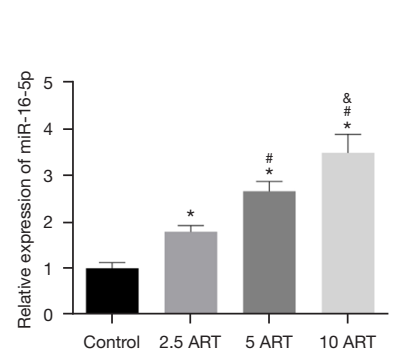

$\mathrm{H}$

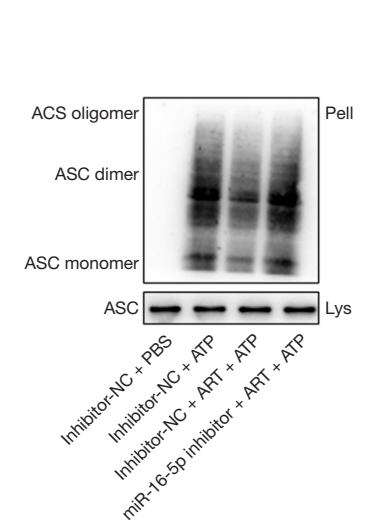

G

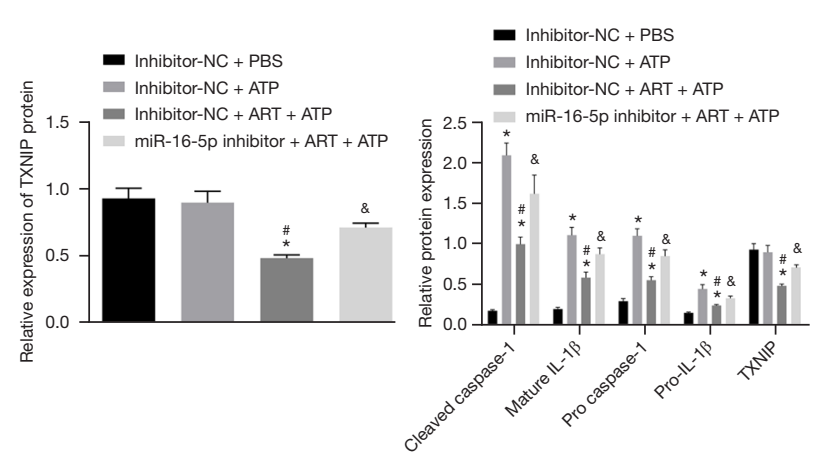

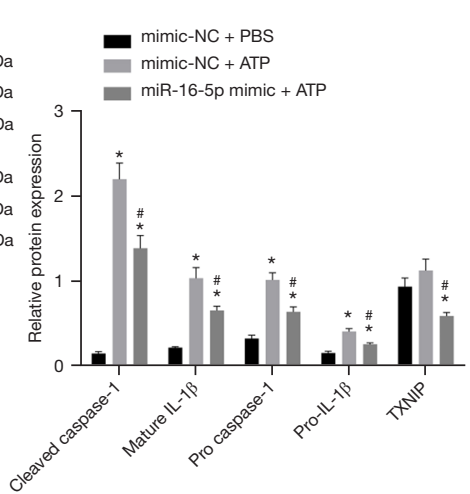

$\mathrm{F}$

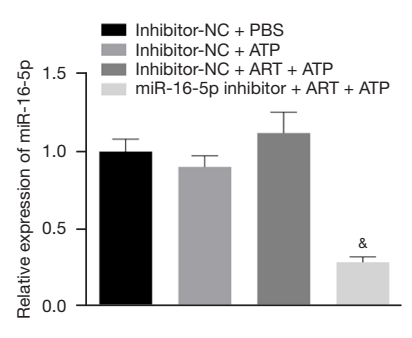

I

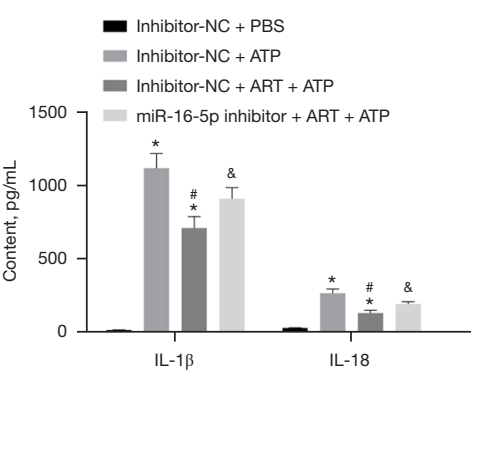

Figure 5 Artesunate blocks the NLRP3 inflammasome via inhibition of miR-16-5p-mediated TXNIP. (A,E,F) The expression of miR-16$5 \mathrm{p}$ in macrophages determined by RT-qPCR. (B,G) The protein expression of TXNIP in macrophages, pro-caspase-1, -IL-1 $\beta$ and -IL18 and their mature forms released in macrophages assessed by immunoblotting. (C,I) The expression of IL-1 $\beta$ and IL-18 in macrophages culture supernatants assessed by ELISA. (D,H) ASC production in macrophages determined by ASC. * (relative to mimic-NC + PBS) and " (relative to mimic-NC + ATP) indicates $\mathrm{P}<0.05$ in panel (A-D); * (relative to control), " (relative to $2.5 \mu \mathrm{mol} / \mathrm{L}$ artesunate), and ${ }^{\text {\& }}$ (relative to $5 \mu \mathrm{mol} / \mathrm{L}$ artesunate) indicates $\mathrm{P}<0.05$ in panel (E); ${ }^{*}$ (relative to inhibitor-NC + PBS), ${ }^{*}$ (relative to inhibitor-NC + PBS), and ${ }^{*}$ (relative to inhibitor-NC + artesunate + ATP) indicates $\mathrm{P}<0.05$ in panel (F-I). Statistical significance was confirmed by one-way ANOVA/Tukey's test. RT-qPCR, reverse transcription quantitative polymerase chain reaction; IL-1 $\beta$, interleukin-1 $\beta$; enzyme-linked immunosorbent assay; ANOVA, analysis of variance; ATP, adenosine triphosphate; ASC, apoptosis-associated speck-like protein containing caspase recruitment domain.

monocytes, while upregulated the expression of miR-16-5p in monocytes $(\mathrm{P}<0.05)$. Rats injected with antagomir-16-5p markedly increased the level of IL- $1 \beta$ and IL-18 in rat blood and the mRNA expression of TXNIP in monocytes, while downregulated the expression of miR-16-5p in monocytes $(\mathrm{P}<0.05)$. The blood liquid level in rats were then assessed by ELISA (Figure 6D), which revealed upregulated LDL, TC, and TG, while lower HDL level in AS rats compared 


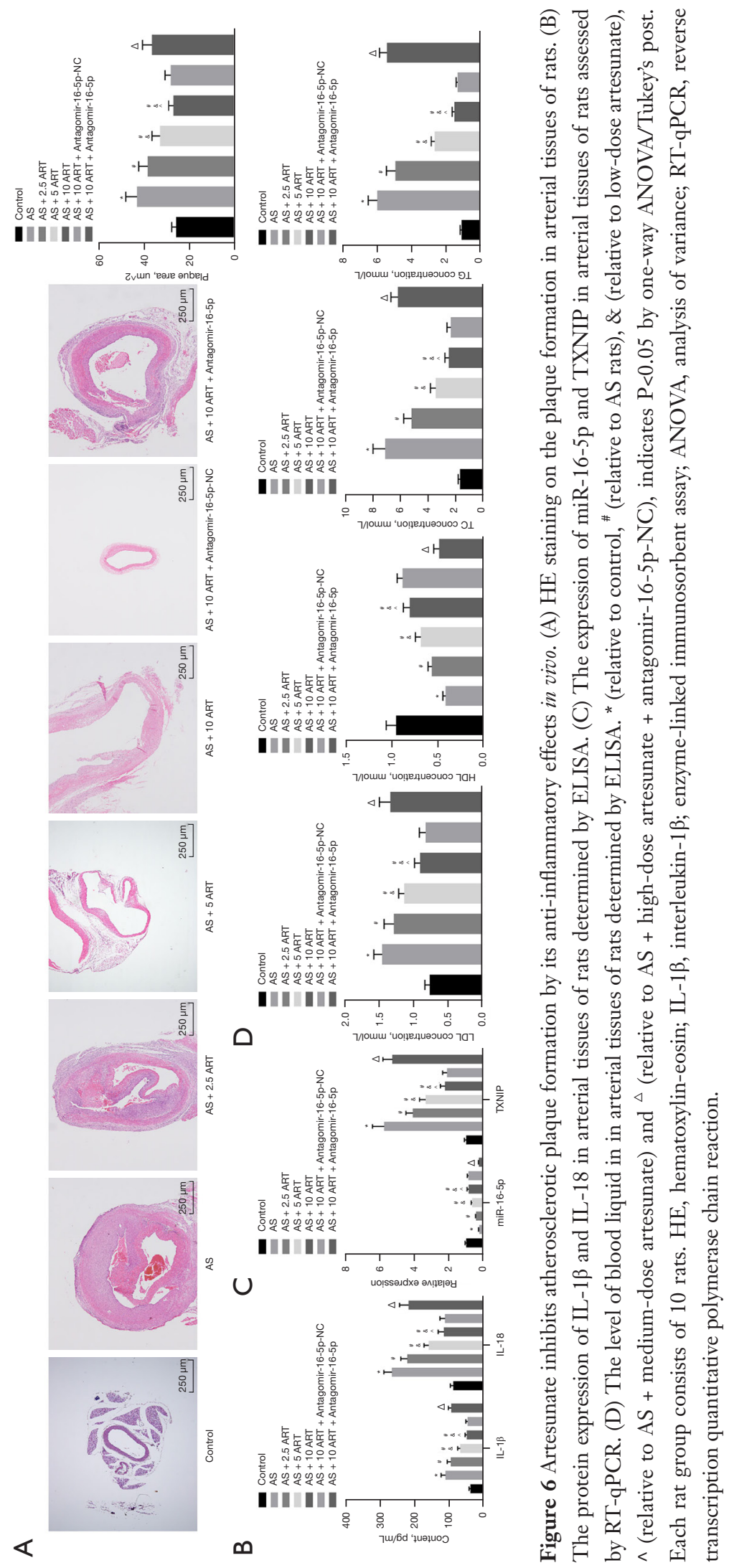


to normal rats, and an opposite trend was observed in AS rats treated with low-dose artesunate, medium-dose artesunate, or high-dose artesunate compared to AS rats. Meanwhile, artesunate treatment at higher concentration strikingly repressed levels of LDL, TC, and TG, while elevated HDL levels. Rats injected with antagomir-16-5p displayed potently upregulated levels of LDL, TC, and TG, while showing downregulated HDL levels. Coherently, artesunate at higher concentration exhibited the antiinflammatory effect by downregulating TXNIP via miR16-5p upregulation evidenced by reduced level of IL-1 $\beta$ and IL-18 in rat blood, LDL, TC, and TG, but elevated HDL level in blood liquid level, which was reversed by inhibition of miR-16-5p in vivo.

\section{Discussion}

Inflammation is characteristic of AS and the inflammatory processes occurring in advanced plaques could trigger acute cardiovascular events (18). The formation of plaques could be initiated by leukocyte accumulation while most leukocytes in plaque consist of macrophages, and macrophages are thought to be differentiated from monocytes in circulating blood (19). Artesunate has previously been reported to successfully relieve airway inflammation in allergic asthma (20). Thus, the current study aimed to study the molecular mechanism of how artesunate exhibited anti-inflammatory effect on attenuating AS. We found evidence to support our hypothesis that artesunate inactivated TXNIP-dependent NLRP3 inflammasome by miR-16-5p elevation, which plays an antiinflammatory role on AS.

Initially, we detected repressed miR-16-5p and elevated TXNIP in the PB monocytes of CHD patients, and repressed miR-16-5p or upregulated TXNIP in PB monocytes was correlated with severity of coronary AS evidenced by elevated protein expression of IL- $1 \beta$ and IL18 in PB monocytes of CHD patients. The implication of IL-1 $\beta$ and IL-18 in the inflammatory process was proposed to cause plaque progression (21). The expression of miR$16-5 \mathrm{p}$ was also found to downregulate in acute coronary syndrome (22), and miR-16-5p could also modulate genes from AS (15). Bioinformatic analysis in the current study identified that miR-16-5p could inversely target TXNIP, though it was scarcely documented. Markedly upregulated plasma levels of TXNIP were observed in patients with early type 2 diabetes mellitus, and might be a hallmark of subclinical AS in early type 2 diabetes mellitus (23). Moreover, the upregulated expression of TXNIP was proposed to upregulate the levels of 2 inflammatory cytokines (IL-1 $\beta$ and IL-18) in THP1 cells, thereby engaging in the development of AS (24). Conversely, depletion of TXNIP was reported to relieve inflammation of vascular smooth muscle cells, thereby ameliorating AS in apolipoprotein E knockout mice (25).

Furthermore, the activation of NLRP3 inflammasome contributed to the elevated protein expression of IL$1 \beta$ and IL-18, and upregulated caspase- 1 and mature IL- $1 \beta$ and mature IL-18, while artesunate could block NLRP3 inflammasome activation, thereby attenuating AS. The release of caspase- 1 and mature IL-1 $\beta$ was indicative of inflammasome formation in macrophages (26). A prior study noted that artesunate ameliorates diabetic nephropathy by blocking the NLRP3 inflammasome (7) and the inhibitory role of artesunate on NLRP3 was also studied in remote lung inflammation induced by renal ischemia-reperfusion (27). In addition, the inhibition of NLRP3 inflammasome activation attenuated AS, accompanied by inhibited plasma levels of proinflammatory cytokines (28). Interestingly, TXNIP is a protein binding to NLRP3 and TXNIP exerted regulatory role on NLRP3 inflammasome activation both in vivo and in vitro (29). The activation of TXNIP-NLRP3 inflammasome contributed to the secretion of inflammatory cytokines inflammation (30).

In the subsequent in vivo experiment, artesunate at higher concentration or miR-16-5p elevation enhanced the anti-inflammatory effect. The severity of AS was correlated with lipid accumulation in the intima, arterial wall thickening, and the narrowing of vascular cavity, accompanied by endothelial cell dysfunction (31). Consistently, aortic intima-media thickening, AS plaque formation, and endothelial cell destruction were observed in rats treated with artesunate at lower concentration in our in vivo experiment. The accumulation of LDL was indicative of more severe AS (32). The decreased contents of TC and TG, but increased expression of HDL-C was favorable to AS in diabetic rats (33). Consistently, our results revealed that rats treated with artesunate at higher concentrations or antagomir-16-5p-NC exhibited repressed TC and TG, but elevated HDL.

\section{Conclusions}

In conclusion, the current study sheds light on the mechanism underlying the anti-inflammatory effect of artesunate on AS. Particularly, artesunate blocked TXNIP- 


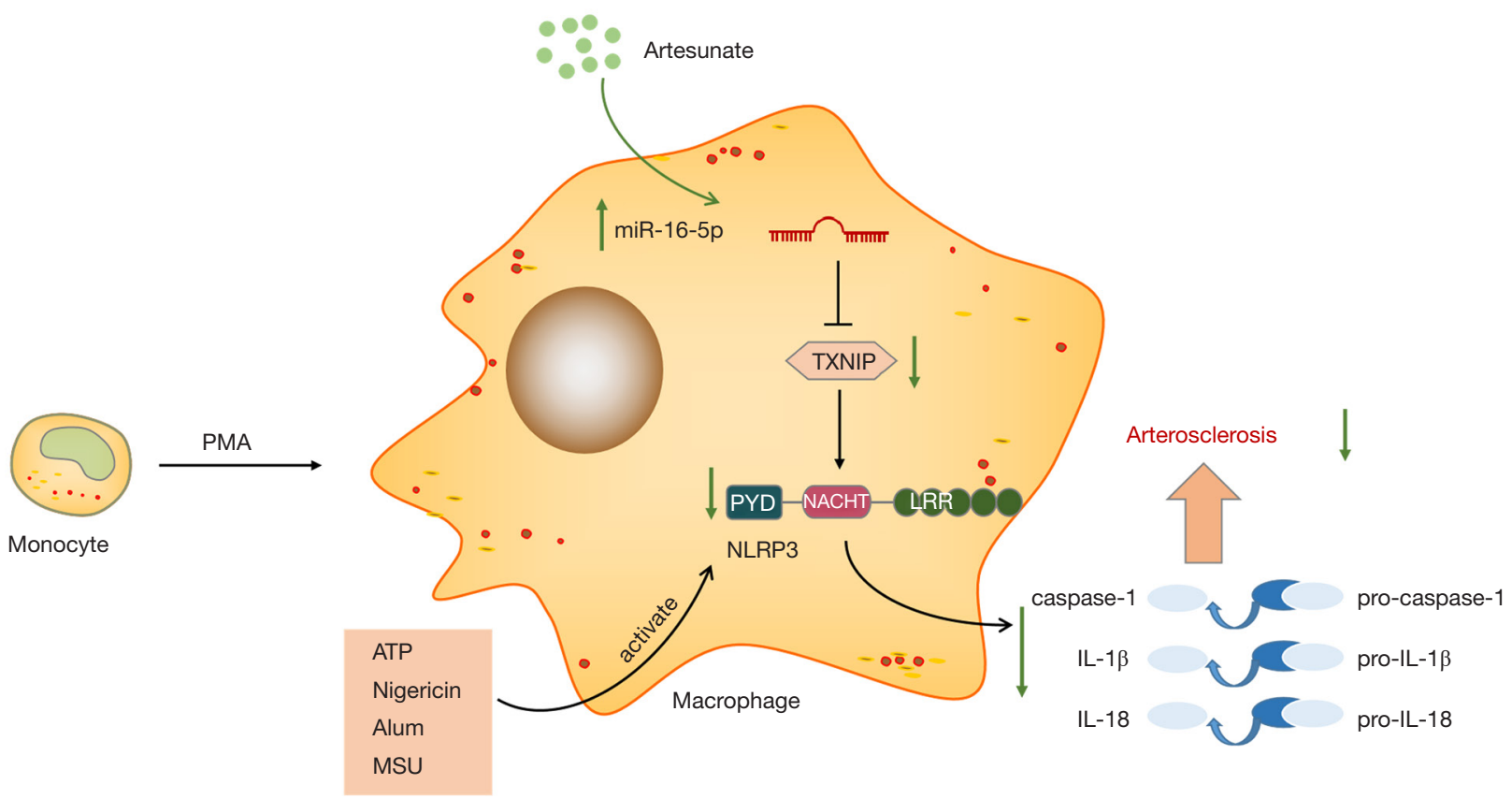

Figure 7 The mechanism graph of the regulatory network and function of artesunate on AS. Artesunate blocks TXNIP-mediated NLRP3 inflammasome via the upregulation of miR-16-5p to exert anti-inflammatory effects on AS.

mediated NLRP3 inflammasome via the upregulation of miR-16-5p (Figure 7). This mechanism helps to better elucidate the molecular mechanism of artesunate on AS, which also makes artesunate a therapeutic target for AS in the future. However, further studies are needed to be conducted on the precise binding form mediating the association between miR-16-5p and TXNIP.

\section{Acknowledgments}

Funding: Presided over a project of the National Natural Science Foundation of China Youth Fund (81800739), with a funding of 210,000 yuan; Presided over a general project of the National Natural Science Foundation of China (82170826), with a funding of 550,000 yuan.

\section{Footnote}

Reporting Checklist: The authors have completed the ARRIVE reporting checklist. Available at https://dx.doi. org/10.21037/atm-21-4939
Data Sharing Statement: Available at https://dx.doi. org/10.21037/atm-21-4939

Conflicts of Interest: All authors have completed the ICMJE uniform disclosure form (available at https://dx.doi. org/10.21037/atm-21-4939). The authors have no conflicts of interest to declare.

Etbical Statement: The authors are accountable for all aspects of the work in ensuring that questions related to the accuracy or integrity of any part of the work are appropriately investigated and resolved. This study was performed under approval by the Ethics Committee in the Fourth Affiliated Hospital of Harbin Medical University and followed the tenets of the Declaration of Helsinki (as revised in 2013). Each individual provided written informed consent prior to their participation. Animal experiments were approved by Animal ethics committee of the Fourth Affiliated Hospital of Harbin Medical University for the care and use of animals. The study was also conducted in strict accordance to the standards of the Guide for the Care 
and Use of Laboratory Animals issued by the National Institutes of Health.

Open Access Statement: This is an Open Access article distributed in accordance with the Creative Commons Attribution-NonCommercial-NoDerivs 4.0 International License (CC BY-NC-ND 4.0), which permits the noncommercial replication and distribution of the article with the strict proviso that no changes or edits are made and the original work is properly cited (including links to both the formal publication through the relevant DOI and the license). See: https://creativecommons.org/licenses/by-nc-nd/4.0/.

\section{References}

1. Schaftenaar F, Frodermann V, Kuiper J, et al. Atherosclerosis: the interplay between lipids and immune cells. Curr Opin Lipidol 2016;27:209-15.

2. Libby P, Okamoto Y, Rocha VZ, et al. Inflammation in atherosclerosis: transition from theory to practice. Circ J 2010;74:213-20.

3. Verweij SL, van der Valk FM, Stroes ES. Novel directions in inflammation as a therapeutic target in atherosclerosis. Curr Opin Lipidol 2015;26:580-5.

4. Hou L, Huang H. Immune suppressive properties of artemisinin family drugs. Pharmacol Ther 2016;166:123-7.

5. Jiang $\mathrm{W}$, Cen $\mathrm{Y}$, Song $\mathrm{Y}$, et al. Artesunate attenuated progression of atherosclerosis lesion formation alone or combined with rosuvastatin through inhibition of proinflammatory cytokines and pro-inflammatory chemokines. Phytomedicine 2016;23:1259-66.

6. Komatsu T, Tanaka Y, Kitagawa Y, et al. Sendai Virus V Protein Inhibits the Secretion of Interleukin- $1 \beta$ by Preventing NLRP3 Inflammasome Assembly. J Virol 2018;92:e00842-18.

7. Sun Z, Ma Y, Chen F, et al. Artesunate ameliorates high glucose-induced rat glomerular mesangial cell injury by suppressing the TLR4/NF- $\kappa$ B/NLRP3 inflammasome pathway. Chem Biol Interact 2018;293:11-9.

8. Baldrighi M, Mallat Z, Li X. NLRP3 inflammasome pathways in atherosclerosis. Atherosclerosis 2017;267:127-38.

9. Zhang X, Zhang JH, Chen XY, et al. Reactive oxygen species-induced TXNIP drives fructose-mediated hepatic inflammation and lipid accumulation through NLRP3 inflammasome activation. Antioxid Redox Signal 2015;22:848-70.
10. Hirata CL, Ito S, Masutani H. Thioredoxin interacting protein (Txnip) forms redox sensitive high molecular weight nucleoprotein complexes. Arch Biochem Biophys 2019;677:108159.

11. Tseng PC, Kuo CF, Cheng MH, et al. HECT E3 Ubiquitin Ligase-Regulated Txnip Degradation Facilitates TLR2-Mediated Inflammation During Group A Streptococcal Infection. Front Immunol 2019;10:2147.

12. Krol J, Loedige I, Filipowicz W. The widespread regulation of microRNA biogenesis, function and decay. Nat Rev Genet 2010;11:597-610.

13. Li Y, Yang N, Dong B, et al. MicroRNA-122 promotes endothelial cell apoptosis by targeting XIAP: Therapeutic implication for atherosclerosis. Life Sci 2019;232:116590.

14. Ren K, Zhu X, Zheng Z, et al. MicroRNA-24 aggravates atherosclerosis by inhibiting selective lipid uptake from HDL cholesterol via the post-transcriptional repression of scavenger receptor class B type I. Atherosclerosis 2018;270:57-67.

15. Assmann TS, Recamonde-Mendoza M, Costa AR, et al. Circulating miRNAs in diabetic kidney disease: case-control study and in silico analyses. Acta Diabetol 2019;56:55-65.

16. Zhao X, Zhang HW, Sun D, et al. Relation of oxidizedlow-density lipoprotein and high-density lipoprotein subfractions in non-treated patients with coronary artery disease. Prostaglandins Other Lipid Mediat 2019;144:106345.

17. Li J, Lei HT, Cao L, et al. Crocin alleviates coronary atherosclerosis via inhibiting lipid synthesis and inducing M2 macrophage polarization. Int Immunopharmacol 2018;55:120-7.

18. Calder PC. The role of marine omega-3 (n-3) fatty acids in inflammatory processes, atherosclerosis and plaque stability. Mol Nutr Food Res 2012;56:1073-80.

19. Woollard KJ, Geissmann F. Monocytes in atherosclerosis: subsets and functions. Nat Rev Cardiol 2010;7:77-86.

20. Luo Q, Lin J, Zhang L, et al. The anti-malaria drug artesunate inhibits cigarette smoke and ovalbumin concurrent exposure-induced airway inflammation and might reverse glucocorticoid insensitivity. Int Immunopharmacol 2015;29:235-45.

21. Martínez GJ, Celermajer DS, Patel S. The NLRP3 inflammasome and the emerging role of colchicine to inhibit atherosclerosis-associated inflammation. Atherosclerosis 2018;269:262-71.

22. Gacoń J, Badacz R, Stępień E, et al. Diagnostic and prognostic micro-RNAs in ischaemic stroke due to carotid 
artery stenosis and in acute coronary syndrome: a fouryear prospective study. Kardiol Pol 2018;76:362-9.

23. Zhao Y, Li X, Tang S. Retrospective analysis of the relationship between elevated plasma levels of TXNIP and carotid intima-media thickness in subjects with impaired glucose tolerance and early Type 2 diabetes mellitus. Diabetes Res Clin Pract 2015;109:372-7.

24. Yue LM, Gao YM, Han BH. Evaluation on the effect of hydrogen sulfide on the NLRP3 signaling pathway and its involvement in the pathogenesis of atherosclerosis. J Cell Biochem 2019;120:481-92.

25. Byon CH, Han T, Wu J, et al. Txnip ablation reduces vascular smooth muscle cell inflammation and ameliorates atherosclerosis in apolipoprotein $\mathrm{E}$ knockout mice. Atherosclerosis 2015;241:313-21.

26. Dautova Y, Kapustin AN, Pappert K, et al. Calcium phosphate particles stimulate interleukin- $1 \beta$ release from human vascular smooth muscle cells: A role for spleen tyrosine kinase and exosome release. J Mol Cell Cardiol 2018;115:82-93.

27. Liu Z, Qu M, Yu L, et al. Artesunate Inhibits Renal Ischemia-Reperfusion-Mediated Remote Lung Inflammation Through Attenuating ROS-Induced Activation of NLRP3 Inflammasome. Inflammation 2018;41:1546-56.

Cite this article as: Li B, Zhang Z, Fu Y. Anti-inflammatory effects of artesunate on atherosclerosis via miR-16-5p and TXNIP regulation of the NLRP3 inflammasome. Ann Transl Med 2021;9(20):1558. doi: 10.21037/atm-21-4939
28. Wang R, Wang Y, Mu N, et al. Activation of NLRP3 inflammasomes contributes to hyperhomocysteinemiaaggravated inflammation and atherosclerosis in apoEdeficient mice. Lab Invest 2017;97:922-34.

29. Zhou R, Tardivel A, Thorens B, et al. Thioredoxininteracting protein links oxidative stress to inflammasome activation. Nat Immunol 2010;11:136-40.

30. Sun X, Jiao X, Ma Y, et al. Trimethylamine N-oxide induces inflammation and endothelial dysfunction in human umbilical vein endothelial cells via activating ROSTXNIP-NLRP3 inflammasome. Biochem Biophys Res Commun 2016;481:63-70.

31. Chen Q, Lv J, Yang W, et al. Targeted inhibition of STAT3 as a potential treatment strategy for atherosclerosis. Theranostics 2019;9:6424-42.

32. MacRitchie N, Maffia P. 'Blow my mind(in)' - mindin neutralization for the prevention of atherosclerosis? Clin Sci (Lond) 2018;132:1509-12.

33. Wang YZ, Yang L, Li CF. Protective effect of atorvastatin meditated by HMGCR gene on diabetic rats with atherosclerosis: An in vivo and in vitro study. Biomed Pharmacother 2018;104:240-51.

(English Language Editor: J. Jones) 
Supplementary

Table S1 Primer sequences for RT-qPCR

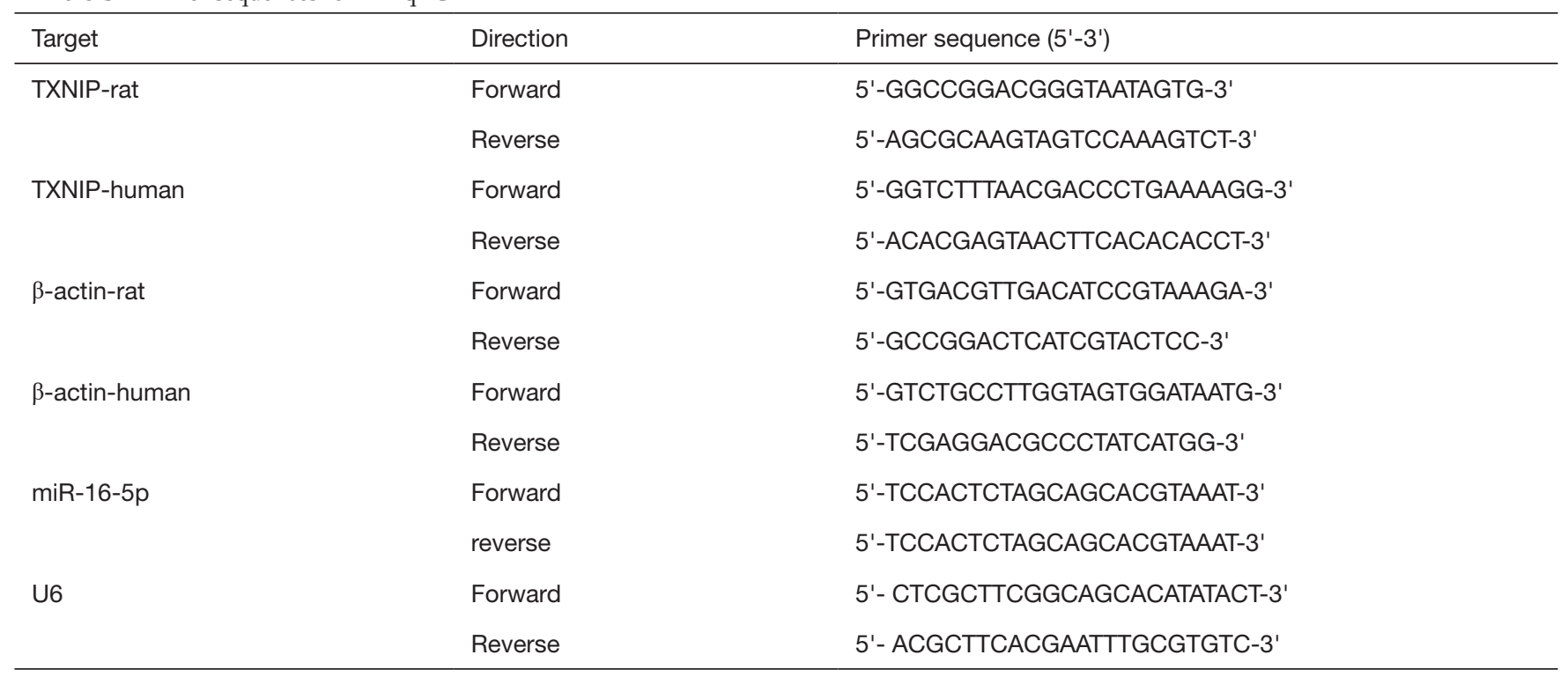

